\title{
INOVAÇÃO \& PROPRIEDADE INDUSTRIAL \& INDÚSTRIA QUÍMICA
}

\author{
Adelaide Maria de Souza Antunes*,\# \\ Instituto Nacional da Propriedade Industrial, Rua Mayrink Veiga, ${ }^{\circ}$ 9, $19^{\circ}$ andar, Rio de Janeiro - RJ, Brasil
}

Recebido em 10/7/13; aceito em 27/8/13; publicado na web em 18/9/13

\begin{abstract}
INNOVATION \& INDUSTRIAL PROPERTY \& THE CHEMICALS INDUSTRY. The main aim of this paper was to contribute to reflections in Brazil on the need to transfer knowledge held at universities and R\&D institutions over to companies, i.e. to transfer scientific knowledge of chemistry to technology. It discusses how the competitiveness of countries is increasingly dependent on their technological capacity. The chemicals industry is a fundamental driver of social, environmental, economic and industrial indicators of sustainable development. In Brazil, the chemicals industry's deficit has grown over the last three decades. Patents are important sources of information because patent documents contain $75 \%$ of all technological information available. The National Institute of Industrial Property in Brazil has created a Technology Observatory with the purpose of identifying and analyzing technological information contained in patent documents within the ambit of partnerships with government entities or business associations, in order to support their technology-related decision-making processes. The paper gives examples of ethanol and biotechnology patent documents, including pharmaceuticals, of which there are very few in Brazil. However, a few of the patent applications identified are filed in Brazil, giving the country the opportunity to transform this scientific knowledge into technology by means of partnership agreements with companies. Finally, the paper presents information on the patent applications filed by the world's leading chemicals companies as measured by their revenues, and the respective numbers of patent applications in the last five years in organic chemistry and polymers, sectors in which Brazil is currently dependent on imports for over $50 \%$ of its needs. The patent assignees in these sectors in Brazil are also identified, and the paper concludes that Brazil needs to invest in the development of professionals, providing clearly-defined career paths in technology innovation teams at R\&D institutions, and to foster more initiatives such as the creation of a new research and innovation entity, EMBRAPII, since investing in science and technology is a prerequisite for knowledge production, industrial property, economic development and, consequently, the competitiveness of the country.
\end{abstract}

Keywords: patents; chemicals industry; competitiveness.

\section{INTRODUÇÃO}

A Química Sem Fronteiras tem como objetivo contribuir para a formulação de Políticas de Ciência e Tecnologia (C\&T) na área da química. ${ }^{1}$ A Diretoria e o Conselho Consultivo da Sociedade Brasileira de Química (SBQ) elaboraram o documento "Eixos Mobilizadores em Química" entre os quais constam aproximações pró-ativas da academia com a atividade econômica e estímulo ao empreendedorismo e à interdisciplinaridade. ${ }^{2}$ Este artigo pretende contribuir para a reflexão no Brasil sobre a necessidade de transferir conhecimento gerado nas universidades e centros de pesquisa e desenvolvimento para as empresas químicas.

A indústria química é meio para as diferentes atividades da economia, suporta a agroindústria por meio dos fertilizantes e dos defensivos agrícolas. Na atividade extrativa se faz presente nos minerais, petróleo e na própria siderurgia. $\mathrm{Na}$ indústria de transformação a química é a essência da petroquímica, da farmacêutica, dos cosméticos e produtos de limpeza além de contribuir nos materiais da construção civil e ser a grande fornecedora do setor têxtil.

O Programa Brasil Maior lançado em agosto de 2011 tem como ações fortalecer a competitividade, acelerar ganhos de produtividade, promover o adensamento produtivo e tecnologia das cadeias de valor, ampliar mercados, criar empregos de melhor qualidade e garantir um crescimento inclusivo e sustentável. ${ }^{3}$ Por outro lado o documento publicado pela Organização Mundial de Propriedade Intelectual (OMPI) em 2011, intitulado The Changing Face of Innovation traz como premissa a inovação como driver central para o crescimento

*e-mail: adelaide@eq.ufrj.br

"Prof ${ }^{a}$. Emérita da Universidade Federal do Rio de Janeiro econômico, para o desenvolvimento e melhoraria de empregos, o que significa desenvolver capacidade, gerar conhecimento. ${ }^{4}$

No entanto, para que ocorra o desenvolvimento econômico e, com o mesmo, a competitividade, faz-se necessário a tecnologia. Segundo a Organização para Cooperação e Desenvolvimento Econômico (OCDE), o dinamismo de um país pode ser medido pelo número de depósitos de patentes. O escopo e o nível de detalhe requerido para proteção de um documento de patente significa que $75 \%$ de toda documentação técnica produzida no mundo está publicada somente nos documentos de patentes. ${ }^{5}$

O Brasil, segundo o Relatório Global de Competitividade, passou do $64^{\circ}$ lugar em 2008 para o $48^{\circ}$ lugar em 2012, no entanto bem abaixo dos países denominados desenvolvidos. Os países que estão, nos últimos dois anos, nos primeiros 4 lugares são Suíça, Singapura, Finlândia e Suécia. ${ }^{6}$

Já segundo o IMD World Competitiveness Yearbook (WCY) O Brasil saiu do $46^{\circ}$ em 2011 para o 51 ${ }^{\circ}$ lugar em 2012. ${ }^{7}$ Estatísticas a parte, o fato é que os países são cada vez mais avaliados, não somente por critérios econômicos, como crescimento do PIB, mas também pela capacidade gerencial, infraestrutura tecnológica e exportação de bens de maior densidade tecnológica, o que reflete a inovação dos países.

No que diz respeito à Indústria Química, segundo a Associação Brasileira da Indústria Química, ABIQUIM ${ }^{8}$ o faturamento líquido registrado em 2012 alcançou US\$ 153 bilhões, sendo 46,5\% deste faturamento de produtos químicos de uso industrial. Os segmentos de maior adensamento químico, como defensivos agrícolas e produtos farmacêuticos, correspondem a somente $6 \%$ e 16,6\% do faturamento, respectivamente. Ou seja, estes dois setores correspondem a menos de 1/4 do faturamento do complexo químico.

Em termos de importação, o complexo químico importou em 2012 
US\$ 43 bilhões, sendo que os produtos químicos de uso industrial são responsáveis por $64 \%$ do total. As exportações no ultimo ano somam US $\$ 14,8$ bilhões, gerando déficit de US\$ 28 bilhões, mostrando a dependência de produtos considerados básicos. Isso significa que há falta de investimentos e, por consequência, falta de desenvolvimento econômico, uma vez que a química perpassa inúmeros setores da economia.

Em pesquisa realizada junto à sociedade e a academia, na Europa, sobre os progressos esperados no campo da ciência e tecnologia para o século XXI, encontra-se em primeiro lugar a cura do câncer, seguido da erradicação da AIDS, diagnóstico genético, hidrogênio como vetor energético e veículo todo de plástico. Isto significa a enorme dependência da química na construção do futuro desejado, quer no desenvolvimento de medicamentos, na busca por combustíveis limpos e na produção de resinas para bens manufaturados mais leves.

\section{DESAFIOS}

Feitas estas considerações, o que se deseja é o mundo melhor, o que implica em desenvolvimento sustentável. Para tanto a inovação é o processo que permite gerar soluções para os desafios sociais e econômicos, e a chave para gerar empregos e para que as firmas compitam globalmente.

Segundo o Indicators of Sustainable Development: Guidelines and Methodologies (IMI) ${ }^{9}$ os indicadores de desenvolvimento sustentável podem ser divididos em quatro grandes vetores: social, econômico, ambiental e sustentável. A Figura 1 apresenta os indicadores de desenvolvimento sustentável.

$\checkmark$ Social
- Saúde (Prevenção e tratamento)
- Habitação
- Suprimento de água
$\checkmark$ Ambiental
- Biodiversidade
- Biotecnologia
- Uso sustentável das fontes
naturais

\section{Econômico}

- Energia (geração)

- Gestão de Resíduos

- Industria de Extração

$\checkmark$ Industrial
- Capacitação
- C\&T
- Informação
- Cooperação

Fonte: Elaboração Própria a partir de dados do Indicators of Sustainable Development: Guidelines and Methodologies

Figura 1. Indicadores de Desenvolvimento Sustentável ${ }^{9}$

No que diz respeito à indústria química, pode-se concluir que a mesma está presente claramente nos vetores social, ambiental e econômico. A chave do vetor industrial é o conhecimento, pois, em termos de indicadores de desenvolvimento sustentável, é medida da capacitação, da ciência \& tecnologia, da informação e da cooperação existente em um país.

No Brasil, o conhecimento está principalmente localizado nas universidades e nas instituições de pesquisa. Estas são consideradas fundamentais para o Sistema Nacional de Inovação. Neste sentido as políticas públicas têm que ter a função de encorajar a comercialização do conhecimento científico.

A competitividade é medida por dados estatísticos como PIB, PIB per capita, P\&D, capacitação de recursos humanos, exportação de produtos e serviços e por indicadores como IDH (que expressa qualidade de vida), IDT (índice de desenvolvimento tecnológico, número de patentes) e GINI (concentração de renda).

O Gráfico 1 mostra que o Brasil vem melhorando em termos de IDH, porém na Figura 2 a concentração de renda ainda deixa a desejar apesar da grande melhoria na última década, caindo de 0,5832 para 0.5448 de 1992 à $2009 .^{10}$

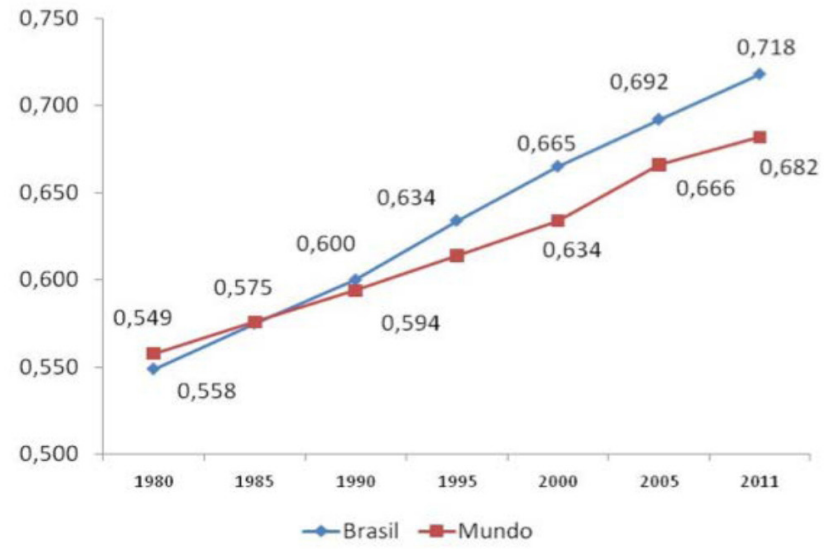

Fonte: Blog Planalto Central do Brasil apud PNUD $2012^{11}$

Gráfico 1. Evolução do IDH do Brasil: 1980-201111

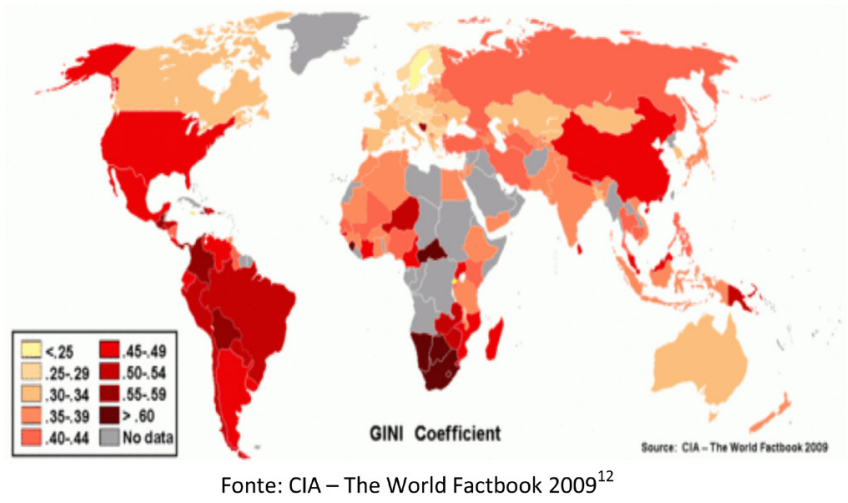

Figura 2. Coeficiente GINI - Mundo ${ }^{I 2}$

Para desenhar o futuro da C\&T utiliza-se a prospecção tecnológica, sendo as informações contidas no depósito de patentes o método mais frequente em estudos prospectivos no mundo por parte de empresas e órgãos governamentais. ${ }^{13}$

As atividades da economia com maior número de depósitos de patentes em 2012 foram: área de computação e periféricos, telecomunicações, automotiva, semicondutores, farmacêutica, dispositivos médicos, bens de consumo, aeroespecial, biotecnologia, petróleo, alimentos e cosméticos. Pode-se verificar que várias áreas têm a química como fornecedora de insumos. ${ }^{14}$

As Figuras 3, 4 e 5 apresentam, respectivamente, o número de depósitos dos últimos anos das principais empresas dos setores de petróleo, química em geral e higiene pessoal. Cabe observar que existe o período de sigilo para publicação do depósito de patente, o que acarreta no atraso de indexação nas bases de dados de patentes.

\section{PROPRIEDADE INDUSTRIAL E INOVAÇÃO}

No documento supracitado da OMPI ${ }^{4}$ é detectado que as estatísticas de patentes nacionais dos países intitulados BRIC (Brasil, Rússia, Índia e China) apresentam um significativo número de depósitos das universidades chinesas, seguida das do Brasil e de instituições de pesquisa da Índia.

A propriedade industrial ocorre também por meio de alianças na Pesquisa e Desenvolvimento, mais visíveis em áreas como a biotecnologia, na indústria química e na tecnologia de informação e comunicação. Portanto, a inovação está atrelada a gestão da P\&D.

Nos países desenvolvidos, os detentores de propriedade industrial estão focados nas estratégias de negócios. No mundo, o número de depósitos de patentes aumentou substancialmente, passando de 
Evolução das patentes da Exxon

Mobil

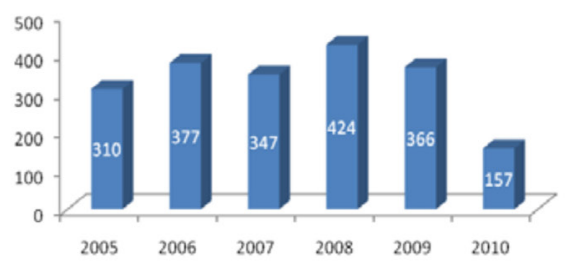

Evolução das patentes da Chevron

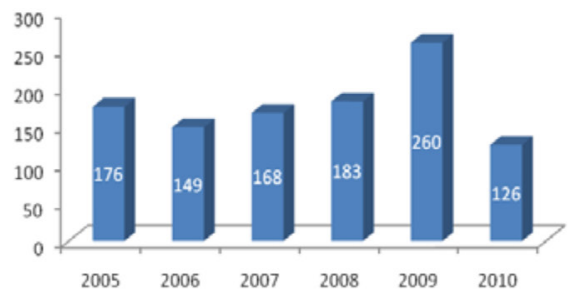

Evolução das patentes da Conoco Phillips

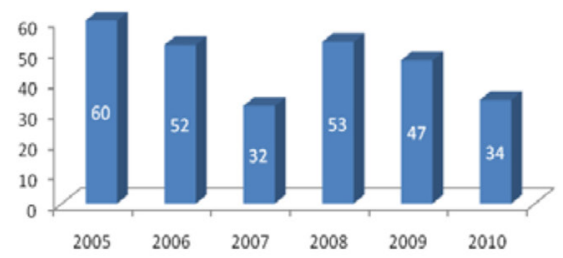

Fonte: Elaboração SIQUIIM ${ }^{1}$ a partir de dados da base Derwent Innovation Index ${ }^{1}$ Sistema de Informação sobre a Industria Química / Escola de Quimica / Universidade Federal do Rio de Janeiro

Figura 3. Número de depósitos das principais empresas de petróleo

800.000 em 1980 para 1,8 bilhão em 2009, sendo que na década de 80 o maior crescimento dos depósitos foi do Japão, já nos anos 90 foram dos Estados Unidos, da Europa e da Coreia e na primeira década do século XXI a China se destaca em termos de depósito de patentes. ${ }^{4}$

Observa-se também maior dinâmica entre os escritórios de transferência de tecnologia nas universidades e organizações de pesquisa pública no mundo. $\mathrm{O}$ aumento de depósitos de patentes demonstra a inovação acumulativa.

A propriedade industrial deve contribuir para a construção e manutenção do ambiente de negócios favorável à inovação, que ofereça informação e conhecimento, promova e proteja investimentos, favoreça a cooperação entre os diferentes atores dos sistemas locais, nacionais e transnacionais de inovação.

O Instituto Nacional de Propriedade Industrial (INPI) criou recentemente o Observatório Tecnológico (OBTEC), que tem como objetivo desenvolver estudos, diagnósticos e pesquisas focadas e contínuas de maneira colaborativa entre o INPI e parceiros com prioridade para as áreas de saúde, defesa, energia, biotecnologia e tecnologia assistivas. ${ }^{15}$

Neste sentido já firmou parceira com a FINEP e a FIOCRUZ e está em vias de assinar parceria com a ABIQUIM. O OBTEC identifica as tecnologias disponíveis, isto é, não protegidas no país, como:

- Patentes brasileiras expiradas - domínio público (ou em vias de);

- Patentes que caducaram por abandono;

- Tecnologias não patenteadas no Brasil.
Evolução das Patentes da Dow Chem

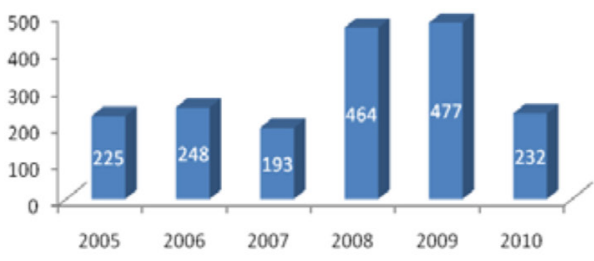

Evolução das patentes da Dupont

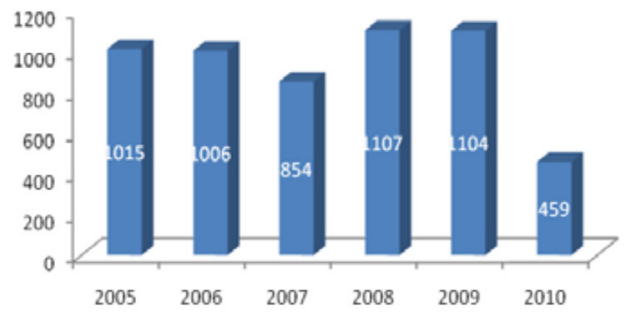

Evolução das patentes da PPG Industries

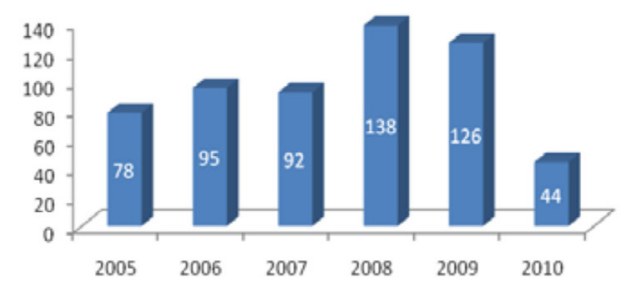

Fonte: Elaboração SIQUIM, a partir de dados da base Derwent Innovation Index

Figura 4. Número de depósitos das principais empresas químicas

Tomando como exemplo o bioetanol, enquadrado pelo governo como um programa em que o país tem que consolidar e expandir a liderança. Observa-se a partir da busca na base de patentes Derwent Innovation Index, disponível através do Portal Capes, utilizando como estratégia no título a palavra "etanol" entre 2005 e 2010, que os principais depositantes de patentes são China e Japão, conforme apresentado na Tabela 1.

Portanto, se etanol é um programa em que o Brasil quer mostrar liderança, e se o mesmo tem tecnologia, precisa saber protegê-la.

Outro exemplo é apresentado no Gráfico 2 a respeito do número de depósitos de Biotecnologia no Brasil, nos Estados Unidos e na Europa e o total de depósitos no mundo no período de 2000 a 2010. Observa-se o pequeno número de depósitos no país e que a grande maioria dos documentos de patentes não foram protegidos no Brasil.

Em outro estudo, pode-se verificar que conjugando a classificação internacional de patentes para biotecnologia $(\mathrm{C} 12 \mathrm{~N})$ e a de formulação médica (A61K) para os últimos 5 anos (2005-2010), o perfil dos depositantes predominantes no mundo são universidades e centros de $\mathrm{P} \& \mathrm{D}$, e da mesma maneira no Brasil se destacam as universidades, conforme demonstrado nas Tabelas 2 e 3 a seguir.

Em consulta à NASDAQ (Stock Market American Exchange), ${ }^{16}$ bolsa de valores eletrônica que lista mais de 2.800 ações de pequena e médica capitalização, as empresas mais comuns estão nas áreas de eletrônica, telecomunicações e biotecnologia, ou seja, empresas de maior densidade tecnológica. As empresas que mais tiveram ganho na 


\section{Evolução das patentes da Procter \&}

Gamble

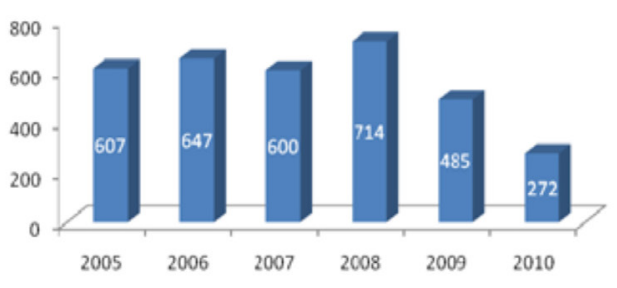

Evolução das patentes da Kimberly

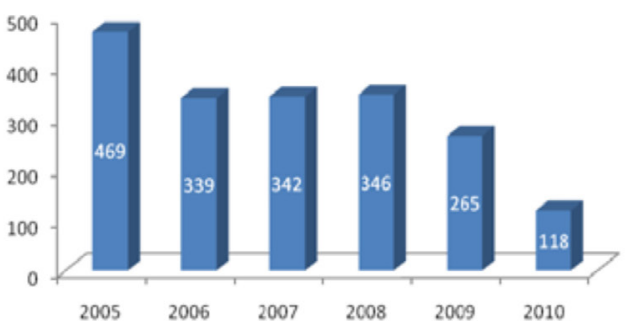

Evolução das patentes da Colgate

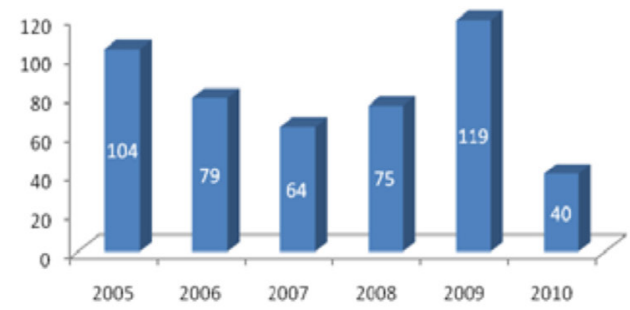

Fonte: Elaboração SIQUIM, a partir de dados da base Derwent Innovation Index

Figura 5. Número de depósitos das principais empresas de higiene pessoal

Tabela 1. Principais depositantes no Mundo sobre Etanol

\begin{tabular}{|c|c|c|}
\hline \multicolumn{3}{|c|}{ ETANOL } \\
\hline Total de Patentes & & 6.465 \\
\hline Total de Diferent & Depositantes & 3.077 \\
\hline \multicolumn{3}{|c|}{ Principais Depositantes } \\
\hline $\mathrm{N}^{\mathrm{o}}$ de Patentes & Depositantes & País \\
\hline 48 & Univ Tianjin & China \\
\hline 44 & Univ Zhejiang & China \\
\hline 43 & Kao Corp & Japão \\
\hline 33 & Cosmo Oil Co Ltd & Japão \\
\hline 31 & Toyota Jidosha Kk & Japão \\
\hline 29 & Tianjin Tianshili Pharm Co Ltd & China \\
\hline 26 & Du Pont De Nemours \& Co E I & EUA \\
\hline 26 & Univ Qinghua & China \\
\hline 25 & Lion Corp & Japão \\
\hline 23 & Honda Motor Co Ltd & Japão \\
\hline
\end{tabular}

Fonte: Elaboração SIQUIM, a partir de dados da base Derwent Innovation Index.

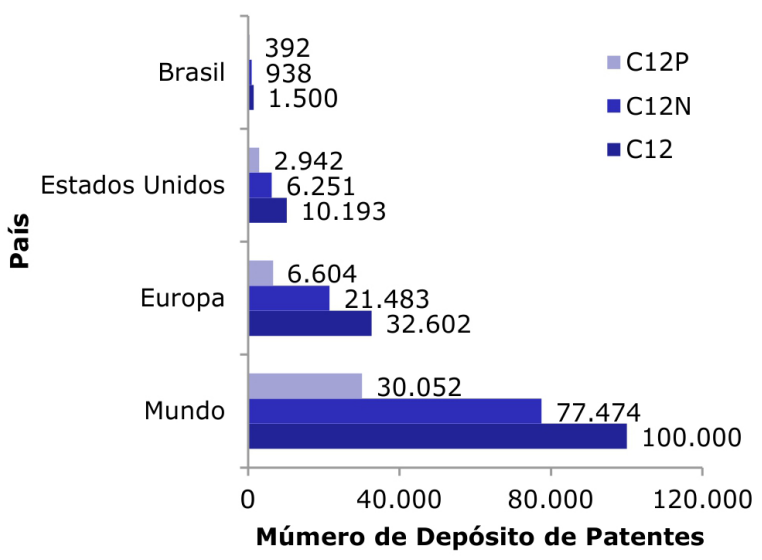

Fonte: Elaboração SIQUIM, a partir de dados da base Derwent Innovation Index

Gráfico 2. Número de depósito de patentes por país por classificação. De acordo com a Classificação Internacional de Patentes: $C 12 P$ relativo a processos de fermentação e enzimáticos; $C 12 \mathrm{~N}$ relativo a microorganismos ou enzimas; $\mathrm{C} 12$ relativo à biotecnologia em geral

Tabela 2. Principais depositantes em Biotecnologia e Farmacêutica no Mundo no período de 2005 a 2010

\begin{tabular}{lc}
\hline \multicolumn{1}{c}{ Depositantes } & $\begin{array}{c}\text { Número de } \\
\text { Depósitos }\end{array}$ \\
\hline Universidade da California & 320 \\
US Dept Health \& Human Services & 288 \\
Novartis Ag & 266 \\
Genentech Inc & 244 \\
Wyeth & 182 \\
CNRS - Cent Nat Rech Sci & 174 \\
Universidade de Tóquio & 165 \\
Inserm - Inst Nat Sante \& Rech Medicale & 156 \\
Universidade do Texas & 155 \\
Merck \& Co Inc & 153 \\
\hline
\end{tabular}

Fonte: Elaboração SIQUIM, a partir de dados da base Derwent Innovation Index.

Tabela 3. Principais depositantes em Biotecnologia e Farmacêutica no Brasil no período de 2005 a 2010

\begin{tabular}{lc}
\hline Depositantes - com Prioridade no Brasil (80) & $\begin{array}{c}\text { Número de } \\
\text { Depósitos }\end{array}$ \\
\hline Universidade Federal de Minas Gerais & 18 \\
Fundação Oswaldo Cruz & 11 \\
Universidade de São Paulo & 8 \\
Universidade Federal do Estado do Rio de Janeiro & 6 \\
Genoa Biotecnologia Sa & 4 \\
Universidade Estadual de Campinas & 3 \\
Universidade Fundação Brasília & 3 \\
Biolab Sanus Farm Ltda & 2 \\
Fundacao Butantan & 2 \\
Farmacore Biotecnologia Ltda & 2 \\
Universidade Federal do Rio de Janeiro & 2 \\
\hline
\end{tabular}

Fonte: Elaboração SIQUIM, a partir de dados da base Derwent Innovation Index. 
Tabela 4. Principais Depositantes em Química Orgânica no Mundo

\begin{tabular}{|c|c|c|c|}
\hline Depositante & $\mathrm{N}^{\mathrm{o}}$ de patentes & Posição na Global 500 - 2012 & Faturamento US\$milhão - 2012 \\
\hline Basf Se (Alemanha) & 877 & $62^{\circ}$ & 102.194 \\
\hline Sumitomo Chemical Co (Japão) & 774 & $191^{\circ}$ & 49.967 \\
\hline China Petrochemical Co Ltd (China) & 648 & N.d. & \\
\hline Bayer (Alemanha) & 638 & $187^{\circ}$ & 50.79 \\
\hline Universidade de ZHEJIANG (China) & 600 & N.d. & \\
\hline Hoffmann La Roche\&Co Ag (Suíça) & 548 & $192^{\circ}$ & 49.714 \\
\hline Du Pont De Nemours\&CO (EUA) & 474 & $270^{\circ}$ & 38.719 \\
\hline Fuji Film Corp (Japão) & 422 & $400^{\circ}$ & 27.804 \\
\hline Monsanto Technology Llc (EUA) & 375 & N.d. & \\
\hline Dow Global Technologies LLC (EUA) & 350 & $156^{\circ}$ & 59.985 \\
\hline
\end{tabular}

Fonte: Elaboração própria a partir de dados da base Derwent Innovation Index utilizando a IPC C07 (Química Orgânica).

Tabela 5. Principais Depositantes em Polímeros no Mundo

\begin{tabular}{lccc}
\hline Depositante & $\mathrm{N}^{\circ}$ de patentes & Posição na Global 500 - 2012 & Revenue \$ million - 2012 \\
\hline Fuji Film Corp (Japão) & 1184 & $400^{\circ}$ & 27.804 \\
Sumitomo Chemical Co (Japão) & 1130 & $191^{\circ}$ & 49.967 \\
BASF Se (Alemanha) & 1081 & $62^{\circ}$ & N.d. \\
Toray Industries (Japão) & 782 & $156^{\circ}$ & 59.194 \\
Dow Global Technologies LLC (EUA) & 693 & $270^{\circ}$ & 38.719 \\
Du Pont de Nemours \& CO (EUA) & & N.d. \\
Asahi Kasei Pharma Corp (Japão) & 682 & N.d. \\
JSR Corp (Japão) & 670 & N.d. \\
Kaneka Corp (Japão) & 650 & 648 & $276^{\circ}$ \\
Bridgestone Corp (Japão) & 645 & & 37.943 \\
\hline
\end{tabular}

Fonte: Elaboração própria a partir de dados da base Derwent Innovation Index utilizando a IPC C08(Compostos macromoleculares orgânicos; sua preparação ou seu processamento químico; composições baseadas nos mesmos).

NADASQ, no primeiro período de 2013, são as empresas de pequeno porte de base biotecnológica e/ou farmacêutica, novamente mostrando a importância da química para o desenvolvimento tecnológico.

\section{PRINCIPAIS DETENTORES DE PATENTES DA INDÚSTRIA QUÍMICA}

No ultimo triênio (2010-2013) observa-se que as empresas químicas com maior número de depósitos estão na sua maioria entre os 500 global players em termos de faturamento. As Tabelas 4 e 5 apresentam, respectivamente, os líderes de depósitos considerando a química orgânica (classificação internacional de patentes - IPC - C07) $)^{17}$ e os líderes na área de polímeros (IPC - C08). ${ }^{17}$ Para cada empresa são explicitados o número de depósitos e a posição no ranking das 500 maiores empresas em termos de faturamento segundo a revista Fortune (Global 500, 2012) $)^{18}$ e o faturamento em 2012.

O Brasil não é visto como ameaça em termos de se apropriar da tecnologia, pois o número de depósitos realizados no país por residentes e não residentes é mínimo, como pode ser visto nas Tabelas 6 e 7.

O aumento da competição pela globalização e novas políticas de regulação induz à rápida mudança técnica tornando fundamental a necessidade de cooperação. A parceria permite dividir riscos e custos e alavancar recursos por consequência aumenta o conhecimento.

Não há outra fórmula para o país que transformar o conhecimento existente nas universidades e nos centros de P\&D em tecnologia. Assim como há incubadoras e parques tecnológicos o Brasil urge
Tabela 6. Principais Depositantes em Química Orgânica no Brasil

\begin{tabular}{lc}
\hline Depositante & $\mathrm{N}^{\circ}$ de patentes \\
\hline Les Lab Servier (França) & 17 \\
Petrobras Petróleo Brasil (Brasil) & 13 \\
Univ Federal Rio de Janeiro (Brasil) & 12 \\
Univ São Paulo (Brasil) & 11 \\
IFP (Brasil) & 10 \\
Univ Estadual Campinas (Brasil) & 9 \\
Rohm \& Haas (EUA) & 8 \\
Fundação Amparo a Pesquisa (Brasil) & 7 \\
Univ Federal Minas Gerais (Brasil) & 7 \\
Bayer (Alemanha) & 6
\end{tabular}

Fonte: Elaboração própria a partir de dados da base Derwent Innovation Index utilizando a IPC C07 (Química Orgânica).

que se tenha, em cada centro gerador de conhecimento, Núcleos de Inovação Tecnológica (NITs) e Empresas de Inovação Industrial.

A formalização da Empresa Brasileira de Pesquisa e Inovação Industrial (EMBRAPII), ${ }^{19}$ em 10 de maio de 2013, para fomentar o processo de cooperação entre pequenas e medias empresas nacionais e instituições tecnológicas ou privadas sem fins lucrativos é uma iniciativa desejada. Os primeiros projetos pilotos envolvem o Instituto de 
Tabela 7. Principais Depositantes em Polímeros no Brasil

\begin{tabular}{lc}
\hline Depositante & $\mathrm{N}^{\circ}$ de patentes \\
\hline Bayer (Alemanha) & 16 \\
Goodyear Tire \& Rubber (EUA) & 14 \\
Xerox Corp (EUA) & 14 \\
Rohm \& Haas (EUA) & 13 \\
Lanxess (Alemanha) & 11 \\
Petrobras Petróleo Brasil (Brasil) & 10 \\
Braskem (Brasil) & 8 \\
Univ Estadual Campinas (Brasil) & 7 \\
Evonik Ind Ag (Alemanha) & 6 \\
Univ Federal Rio de Janeiro (Brasil) & 6 \\
\hline
\end{tabular}

Fonte: Elaboração própria a partir da busca em patentes na base Dewrent Innovation utilizando a IPC C08(Compostos macromoleculares orgânicos; sua preparação ou seu processamento químico; composições baseadas nos mesmos).

Pesquisa Tecnológico (IPT) na área de nanobiotecnologia, o Instituto Nacional de Tecnologia (INT) em energia (gás/petróleo) e saúde e o Centro Integrado de Manufatura e Tecnologia (CIMATEC) do Serviço Nacional de Aprendizagem Industrial (SENAI) na área de automação manufatura. Verifica-se, novamente, que os projetos pilotos têm forte integração com a química.

\section{CONSIDERAÇÕES FINAIS}

Na sociedade moderna a competitividade é baseada no conhecimento e a ciência e a tecnologia são fatores básicos para a geração deste. A inovação depende, portanto, do conhecimento tecnológico.

O artigo mostra que não há praticamente proteção em termos de depósitos de patentes no país na área da química. O conhecimento científico químico tem que ser transformado em tecnologia.

Consultando o Portal de Inovação ${ }^{20}$ e os grupos de pesquisadores cadastrados no $\mathrm{CNPq}$, o Brasil possui inúmeros pesquisadores em quaisquer áreas do conhecimento em que a química esteja envolvida. Possui incubadoras e parques tecnológicos dignos de primeiro mundo. Urge, portanto, que haja mecanismo que agilizem as parcerias entre universidades e empresas. É necessário que sejam destravadas as burocracias nos repasses para as fundações universitárias e que os NITs tenham quadros próprios e com carreiras. Ademais, é fundamental que os depósitos de patentes gerados pelas universidades sejam apresentados às empresas por meio de exposições e feiras.

\section{AGRADECIMENTOS}

À diretoria e ao conselho consultivo da SBQ pelo convite e ao SIQUIM (Sistema de Informação sobre a Indústria Química da Escola de Química da UFRJ) pelo apoio na confecção deste artigo.

\section{REFERÊNCIAS}

1. Pinto, A. C.; Zucco, C.; Galembeck, F.; de Andrade, J. B.; Vieira, P. C; Quim. Nova 2012, 35, 2092.

2. de Andrade, J. B.; Cadore, S.; Vieira, P. C.; Zucco, C.; Pinto, A. C.; Quim. Nova 2003, 26, 445.

3. Plano Brasil Maior: http://www.brasilmaior.mdic.gov.br/, acessada em Setembro 2013.

4. World Intellectual Property Report. The Changing Face of Innovation, 2011.

5. Araujo, V. M. R. H.; Ciência da Informação 1984, 13, 53.

6. Schwab, K; World Economic Forum. The Global Competitiveness Report 2012-2013. Fundação Dom Cabral.

7. IMD World Competitiveness Yearbook: http://www.imd.org/news/ World-Competitiveness-2013.cfm, acessada em Setembro 2013.

8. Associação Brasileira da Indústria Química (ABIQUIM): http://www. abiquim.org.br/, acessada em Setembro 2013.

9. United Nations; Indicators of Sustainable Development: Guidelines and Methodologies, $3^{\text {rd }}$ ed., New York, 2007.

10. CPS/FGV a partir dos microdados da PNAD/IBGE: http://www.sul21. com.br/jornal/2012/03/educacao-e-redistribuicao-de-renda-diminuemdesigualdades-no-brasil/, acessada em Setembro 2013.

11. Blog do Planalto Central: http://www2.planalto.gov.br/, acessada em Setembro 2013.

12. CIA - The World Factbook 2009: https://www.cia.gov/library/ publications/the-world-factbook/, acessada em Setembro 2013.

13. Popper, R.; Global Mapping Report 2007, European Foresight Monitoring Network Report to the EC.

14. Derwent World Patents Index 2012 State of Innovation. Twelve Key Technology Areas. http://img.en25.com/Web/ThomsonReutersScience/1002126.pdf

15. Observatório de Tecnológico: http://www.inpi.gov.br/portal/, acessada em Setembro 2013.

16. NASDAQ Stock Market: http://www.nasdaq.com/, acessada em Setembro 2013.

17. Classificação Internacional de Patentes (IPC): http://ipc.inpi.gov.br/ ipcpub/\#refresh=page, acessada em Setembro 2013.

18. Global 500: http://money.cnn.com/magazines/fortune/global500/, acessada em Setembro 2013.

19. EMBRAPII: http://www.ipt.br/embrapii, acessada em Setembro 2013.

20. Portal de Inovação: http://www.portalinovacao.mcti.gov.br/, acessada em Setembro 2013 